\title{
Effective use of everolimus as salvage chemotherapy for ovarian clear cell carcinoma: a case report
}

This article was published in the following Dove Press journal:

OncoTargets and Therapy

29 January 2014

Number of times this article has been viewed

\section{Eriko Takatori \\ Tadahiro Shoji \\ Yuki Miura \\ Anna Takada \\ Satoshi Takeuchi \\ Toru Sugiyama}

Department of Obstetrics and Gynecology, Iwate Medical University School of Medicine, Morioka, Japan
Correspondence: Tadahiro Shoji Department of Obstetrics and Gynecology, Iwate Medical University School of Medicine, 19-I, Uchimaru, Morioka, Iwate 020-8505, Japan

$\mathrm{Tel}+8 \mathrm{I} 1965 \mathrm{I} 5 \mathrm{III}$

Fax +8I 195221900

Email tshoji@iwate-med.ac.jp
Abstract: A case study using mammalian target of rapamycin complex 1 in recurrent ovarian clear cell carcinoma (CCC) was recently conducted. We report our experience with a patient suffering from recurrent ovarian CCC who achieved long-term disease control with everolimus administration. The patient was a 53-year-old woman who was diagnosed with recurrent ovarian $\mathrm{CCC}$ with dissemination throughout the abdominal cavity. Previously, she had received three chemotherapy regimens, but the disease was progressive and she showed no response to treatment. Therefore, oral everolimus administration (everolimus $10 \mathrm{mg} /$ day on days $1-28$, a 28-day period comprised one cycle) was started. She was administered six cycles. The antitumor response was stable disease, and grade 3 anemia was observed. Chemotherapy was then switched to gemcitabine/docetaxel therapy. In the middle of the second cycle, a rapid increase in ascitic fluid and CA125 elevation were observed. Thereafter, the patient received best supportive care and died of the disease. Everolimus may inhibit malignant progression of ovarian CCC.

Keywords: mTORC1, ovarian cancer, palliative chemotherapy

\section{Introduction}

A case study using mammalian target of rapamycin complex 1 (mTORC1) in recurrent ovarian clear cell carcinoma (CCC) was recently reported in Japan. ${ }^{1}$ Phase II clinical studies in Europe and the United States, using temsirolimus in patients with recurrent ovarian cancer, have also been reported. ${ }^{2}$ In one of these studies, one of three patients with CCC showed a partial response. ${ }^{2}$ Everolimus is a molecular-targeted agent with established efficacy in renal cell cancer. ${ }^{3}$ As the gene expression profiles of ovarian and renal CCCs are reportedly similar, ${ }^{4}$ there is a possibility of everolimus being effective in ovarian CCC that has a molecular background in common with renal CCC. Herein, we report our experience with a patient with recurrent ovarian $\mathrm{CCC}$ who achieved long-term disease control with everolimus administration.

\section{Case report}

The patient was a 53-year-old woman, gravida 3, para 2. She had first menstruated at age 14 years and reached menopause at age 49 years. Her family history and medical history were unremarkable. Due to the diagnosis of ovarian cancer in 2010, she underwent total hysterectomy, bilateral salpingo-oophorectomy, omentectomy, and pelvic and para-aortic lymph node dissection. Adenocarcinoma was diagnosed based on examination of cells from ascitic fluid. The pathological diagnosis of the extracted organ was CCC. Clinically, the ovarian cancer was stage IC. Postoperatively, the 
patient received six cycles of paclitaxel/carboplatin therapy (paclitaxel $175 \mathrm{mg} / \mathrm{m}^{2}$ on day 1 , carboplatin area under the curve $6 \mathrm{mg} / \mathrm{ml}$ per min on day 1, every 21 days) and achieved remission. Within 6 months after the final administration of this chemotherapy, however, the CA125 level increased and ascites developed, while computed tomography (CT) showed dissemination throughout the abdominal cavity. Accordingly, the diagnosis was platinum-resistant recurrent ovarian cancer. Six cycles of CPT-11/pegylated liposomal doxorubicin therapy (CPT-11 $80 \mathrm{mg} / \mathrm{m}^{2}$ on days 1 and 15 , and pegylated liposomal doxorubicin $30 \mathrm{mg} / \mathrm{m}^{2}$ on day 3 , every 28 days) were started in April 2011. Although the antitumor efficacy after three cycles was sufficient to achieve stable disease (SD), progressive disease was apparent at the completion of six cycles. Therefore, the treatment was switched to gemcitabine/docetaxel therapy (gemcitabine $800 \mathrm{mg} / \mathrm{m}^{2}$ on days 1 and 8 , docetaxel $60 \mathrm{mg} / \mathrm{m}^{2}$ on day 8 , every 21 days) starting in November 2011. The patient completed five cycles but did not respond to this therapy, showing progressive disease. After a full explanation to the patient and her husband that there would be no next chemotherapy according to the guidelines for recurrent ovarian cancer and that CCC is always chemotherapy resistant, the patient elected to receive oral administration of everolimus. Oral everolimus administration (everolimus $10 \mathrm{mg}$ /day on days 1-28, a 28-day period comprised one cycle) was started in March 2012 as medical care without health insurance coverage. Figure 1 presents CT images before treatment, after three cycles, and after six cycles. Figure 2 shows the changes in CA125 levels. The tumor reduction rate was $11.4 \%$, according to measurements based on $\mathrm{CT}$ images. The antitumor response according to Response Evaluation Criteria In Solid Tumors (RECIST) 1.1 was SD. ${ }^{5}$ Additionally, the Eastern Cooperative Oncology Group (ECOG) Performance Status ${ }^{6}$ was 1 throughout this therapy. During the sixth cycle, however, the patient discontinued treatment for financial reasons. Treatment was thus suspended upon completion of the sixth cycle. With regard to adverse events, the patient developed grade 3 anemia and grade 1 hypercholesterolemia as hematotoxicities in the fifth and third cycle, respectively. As for non-hematotoxicities, she had grade 1 fatigue, grade 1 malaise, and grade 1 stomatitis. The adverse events in each cycle are shown in Table 1. These adverse events necessitated neither postponement of the start of the next cycle nor suspension of administration. Starting in October 2012, the treatment was again switched to gemcitabine/docetaxel therapy (gemcitabine $800 \mathrm{mg} / \mathrm{m}^{2}$ on days 1 and 8 , docetaxel $60 \mathrm{mg} / \mathrm{m}^{2}$ on day 8 , every 21 days). In the middle of the second cycle, a rapid increase in ascitic fluid

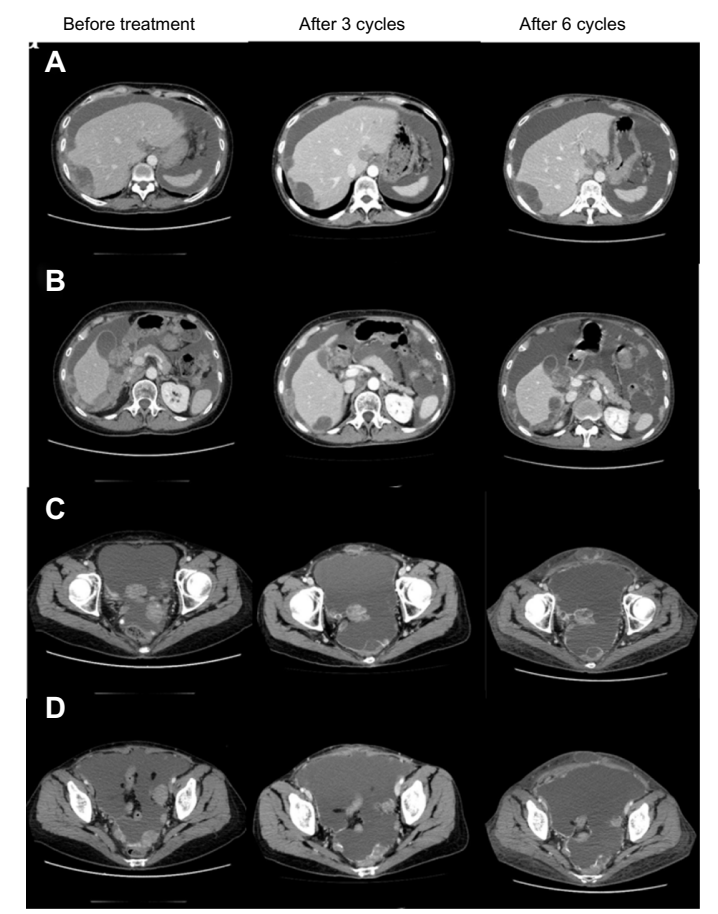

Figure I Computed tomography findings before treatment and after three and six cycles of oral everolimus.

Notes: (A and B) Peritoneal dissemination to the liver surface. (C and D) Peritoneal dissemination to the pouch of Douglas. A large amount of ascitic fluid and widespread peritoneal dissemination are observed. While some reduction of the peritoneal dissemination was seen after three cycles and six cycles of everolimus administration, the overall evaluation of antitumor response was stable disease.

and CA125 elevation were observed. Thereafter, the patient received best supportive care and died in December 2012.

\section{Discussion}

A number of clinical studies using molecular-targeted agents for ovarian cancer have been launched in recent years. GOG0268 is a Phase II clinical study designed to examine additional effects of the mTOR inhibitor temsirolimus when combined with the existing paclitaxel/carboplatin therapy in patients with stages III/IV CCC, whereas GOG0254 is a Phase II clinical study designed to examine the efficacy of the tyrosine kinase inhibitor sunitinib, which is reportedly effective in patients with recurrent or relapsed clear cell adenocarcinoma of the kidney. In addition, clinical studies using olaparib, pazopanib, and AMG386 are also underway. To date, bevacizumab is the only molecular-targeted agent proven to be effective for ovarian cancer. ${ }^{7-9}$

Various genetic characteristics of clear cell adenocarcinoma have been elucidated. Notably, activation of the PI3K (Phosphoinositide 3-kinase) -mTOR-HIF (hypoxia-inducible factor) $-1 \alpha$ pathway is attracting research attention. The mTOR forms mTOR complex (mTORC) 1 and mTORC2 by binding to the regulatory associated protein of mTOR (Raptor) and the rapamycin-insensitive companion of 


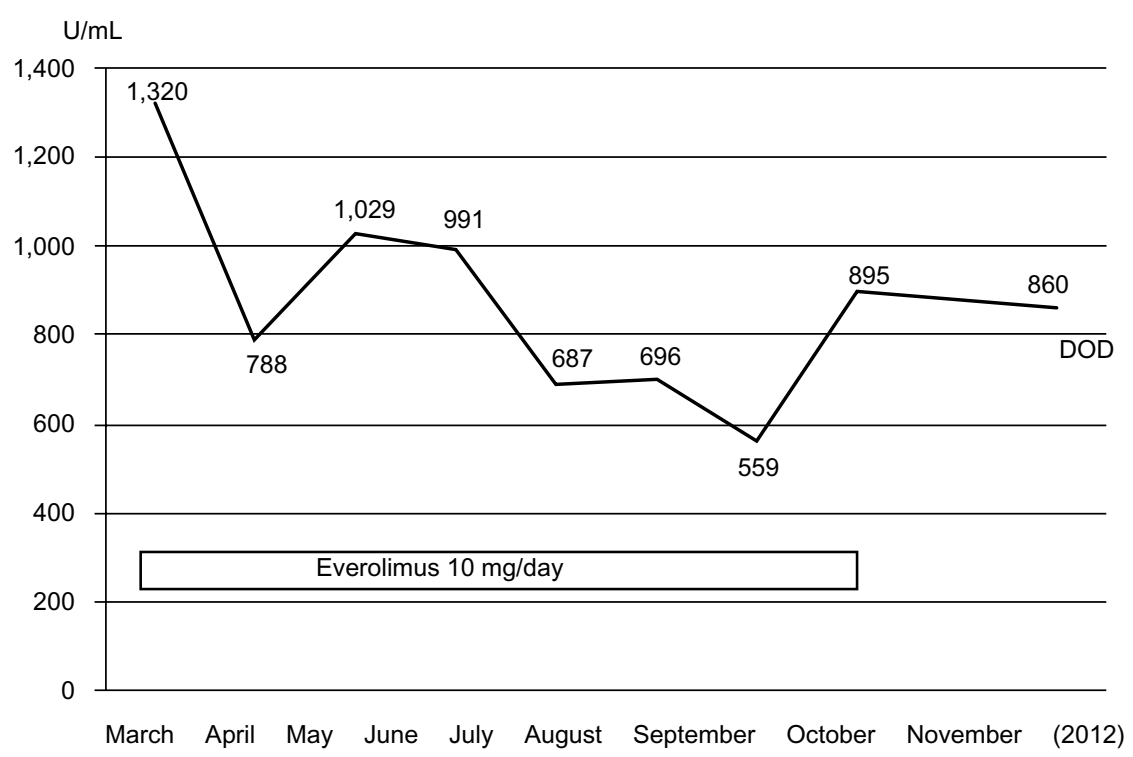

Figure 2 Changes in CAI 25 levels after everolimus administration.

Notes: Serum CAI25 levels started to decrease after the administration of everolimus and then stabilized. The lowest value was $559 \mathrm{U} / \mathrm{mL}$, in September 2012 . Serum CAI 25 levels started to rise after the discontinuation of everolimus.

Abbreviation: DOD, died of disease.

mTOR (Rictor), respectively, and these two complexes have different intracellular functions. The mTORC1 is activated by PI3K-AKT (protein kinase B) and phosphorylates S6K1 and 4EBP1, thereby promoting translation and protein synthesis. The mTORC 2 was only recently identified, and its activation pathway has not yet been elucidated. An activating mutation in PIK3CA, PTEN deletion, and AKT amplification are observed in cancer cells, with the PI3K-AKT-mTORC1 pathway often being over-activated, and this is attracting attention as a potential target of cancer therapy.

The activation rate of AKT-mTORC1 signal is reportedly $50 \%$ in epithelial ovarian cancer. ${ }^{10}$ In particular, the activation rate in CCC is $80 \%$ to $90 \%$, substantially exceeding the $50 \%$ in

Table I Adverse events in each cycle of oral everolimus administration

\begin{tabular}{|c|c|c|c|c|c|c|}
\hline & \multicolumn{6}{|c|}{ Cycle } \\
\hline & I & 2 & 3 & 4 & 5 & 6 \\
\hline & \multicolumn{6}{|c|}{ Grade } \\
\hline Leukopenia & 0 & 0 & 0 & 0 & 0 & 0 \\
\hline Neutropenia & 0 & 0 & 0 & 0 & 0 & 0 \\
\hline Thrombocytopenia & 0 & 0 & 0 & 0 & 0 & 0 \\
\hline Anemia & 2 & 2 & 2 & 2 & 3 & 2 \\
\hline Hypercholesterolemia & 0 & 0 & I & 0 & 0 & 0 \\
\hline Fatigue & I & I & I & 1 & 1 & I \\
\hline Malaise & I & I & I & I & I & I \\
\hline Stomatitis & 0 & 0 & 0 & 0 & I & 0 \\
\hline Dyspnea & 0 & 0 & 0 & 0 & 0 & 0 \\
\hline
\end{tabular}

Note: according to National Cancer Institute Common Terminology Criteria for Adverse Events (version 4.0). ${ }^{20}$ serous adenocarcinoma. PIK3CA gene mutations are observed in approximately $40 \%$ of $\mathrm{CCCs}^{11-13}$ and inactivation of PTEN occurs at a high frequency; ${ }^{14,15}$ these factors are part of the reason for $\mathrm{mTORC} 1$ activation occurring at a high frequency. This also suggests that mTORC1 is a promising therapeutic target for clear cell adenocarcinoma. Moreover, as mTOR inhibitors were confirmed to have antitumor efficacy in vitro and in vivo, ${ }^{1,16}$ their clinical application is anticipated.

Harasawa et al proved that the transcription activity of hypoxia-inducible factor-1 is higher in ovarian clear cell adenocarcinoma than in other histological types, and also demonstrated in vivo that expression of the antitumor effect was significantly higher in the everolimus group than in the control group. ${ }^{17}$ Furthermore, Hirasawa et al compared four groups receiving placebo, everolimus alone, paclitaxel/cisplatin alone, and everolimus + paclitaxel/cisplatin in vivo and reported that antitumor efficacy was highest in the everolimus + paclitaxel/cisplatin group. While tumor growth was inhibited in the group receiving everolimus alone in their report, little cytoreductive effect was obtained..$^{18}$ Therefore, it may be difficult to obtain an antitumor effect in ovarian clear cell adenocarcinoma with everolimus alone, and we anticipate that an additional effect would be obtained by combining this agent with other anticancer drugs.

A Phase II clinical study of everolimus in recurrent or relapsed ovarian CCC is scheduled in Japan. This will be the first clinical study, worldwide, to examine the therapeutic efficacy and safety of everolimus alone for treating clear cell adenocarcinoma. This trial is also designed to serve as 
an exploratory examination of biomarkers for predicting antitumor efficacy.

Interstitial pneumonia is often observed as an adverse reaction to everolimus. In a global Phase III clinical trial (REnal Cell cancer treatment with Oral RAD001 given Daily [RECORD]-1 trial) involving patients with renal cell carcinoma, ${ }^{3}$ interstitial pneumonia occurred in 37 (13.5\%) of 274 patients receiving everolimus. In the CT evaluation by radiologists, interstitial pneumonia newly occurred or showed exacerbation in 132 (53.9\%) of 245 patients, a frequency markedly exceeding that reported by primary physicians. ${ }^{19}$ Blood tests showed hyperglycemia in 50\%, hypercholesterolemia in $76 \%$, and hypertriglyceridemia in $71 \%$ of patients. ${ }^{3}$ Our present patient had no respiratory symptoms or findings of interstitial pneumonia on chest CT at the end of six cycles. While grade 1 hypercholesterolemia was observed during the third cycle, this cholesterol elevation resolved without intervention. Six cycles could be administered safely without serious adverse events, dose reduction, or treatment postponement. While this treatment was discontinued at the completion of the sixth cycle for financial reasons, the antitumor efficacy was demonstrated by CT findings indicating maintenance of SD, and the disease condition showed rapid deterioration immediately after switching from everolimus to gemcitabine/docetaxel therapy. This suggested that antitumor response cannot be obtained by switching to anticancer drugs with different profiles in patients with recurrent ovarian $\mathrm{CCC}$ who are no longer responsive to multiple anticancer drugs and that the molecular-targeted agent everolimus may inhibit malignant progression. The patient might have sustained a progression-free state for a longer time had the everolimus treatment been continued. To our knowledge, this is the first case report, worldwide, describing the use of everolimus in a patient with recurrent ovarian CCC. It is hoped that this report will lead to the listing of everolimus for insurance coverage, as this drug may improve the outcomes of patients with ovarian CCC.

\section{Disclosure}

The authors report no conflicts of interest in this work.

\section{References}

1. Takano M, Kikuchi Y, Kudoh K, et al. Weekly administration of temsirolimus for heavily pretreated patients with clear cell carcinoma of the ovary: a report of six cases. Int J Clin Oncol. 2011;16:605-609.

2. Behbakht K, Sill MW, Darcy KM, et al. Phase II trial of the mTOR inhibitor, temsirolimus and evaluation of circulating tumor cells and tumor biomarkers in persistent and recurrent epithelial ovarian and primary peritoneal malignancies: a Gynecologic Oncology Group study. Gynecol Oncol. 2011;123:19-26.
3. Motzer RJ, Escudier B, Oudard S, et al; RECORD-1 Study Group. Efficacy of everolimus in advanced renal cell carcinoma: a double-blind, randomised, placebo-controlled phase III trial. Lancet. 2008;372:449-456.

4. Zorn KK, Bonome T, Gangi L, et al. Gene expression profiles of serous, endometrioid, and clear cell subtypes of ovarian and endometrial cancer. Clin Cancer Res. 2005;11:6422-6430.

5. EA Eisenhauer, P Therasse, J Bogaerts, LH Schwartz, D Sargent, R Ford, et al. New response evaluation criteria in solid tumours: Revised RECIST guideline (version 1.1). Eur J Cancer. 2009;45:228-247.

6. Oken MM, Creech RH, Tormey DC, Horton J, Davis TE, McFadden ET, et al. Toxicity and response criteria of the Eastern Cooperative Oncology Group. Am J Clin Oncol. 1982;5:649-655.

7. Burger RA, Brady MF, Bookman MA, et al. Incorporation of bevacizumab in the primary treatment of ovarian cancer. $N$ Engl $J$ Med. 2011;365:2473-2483.

8. Perren TJ, Swart AM, Pfisterer J, et al; ICON7 Investigators. A phase 3 trial of bevacizumab in ovarian cancer. N Engl J Med. 2011;365:2484-2496.

9. Aghajanian C, Blank SV, Goff BA, et al. OCEANS: a randomized, double-blind, placebo-controlled phase III trial of chemotherapy with or without bevacizumab in patients with platinum-sensitive recurrent epithelial ovarian, primary peritoneal, or fallopian tube cancer. $J$ Clin Oncol. 2012;30:2039-2045.

10. Altomare DA, Wang HQ, Skele KL, et al. AKT and mTOR phosphorylation is frequently detected in ovarian cancer and can be targeted to disrupt ovarian tumor cell growth. Oncogene. 2004;23:5853-5857.

11. Kuo KT, Mao TL, Jones S, et al. Frequent activating mutations of PIK3CA in ovarian clear cell carcinoma. Am J Pathol. 2009;174: $1597-1601$.

12. Jones S, Wang TL, Shih IeM, et al. Frequent mutations of chromatin remodeling gene ARID1A in ovarian clear cell carcinoma. Science. 2010;330:228-231.

13. Campbell IG, Russell SE, Choong DY, et al. Mutation of the PIK3CA gene in ovarian and breast cancer. Cancer Res. 2004;64:7678-7681.

14. Hashiguchi Y, Tsuda H, Inoue T, Berkowitz RS, Mok SC. PTEN expression in clear cell adenocarcinoma of the ovary. Gynecol Oncol. 2006;101:71-75.

15. Ho CM, Lin MC, Huang SH, et al. PTEN promoter methylation and LOH of 10q22-23 locus in PTEN expression of ovarian clear cell adenocarcinomas. Gynecol Oncol. 2009;112:307-313.

16. Mabuchi S, Kawase C, Altomare DA, et al. mTOR is a promising therapeutic target both in cisplatin-sensitive and cisplatin-resistant clear cell carcinoma of the ovary. Clin Cancer Res. 2009;15:5404-5413.

17. Harasawa M, Yasuda M, Hirasawa T, et al. Analysis of mTOR inhibitioninvolved pathway in ovarian clear cell adenocarcinoma. Acta Histochem Cytochem. 2011;44:113-118.

18. Hirasawa T, Miyazawa M, Yasuda M, et al. Alterations of hypoxiainduced factor signaling pathway due to mammalian target of rapamycin (mTOR) suppression in ovarian clear cell adenocarcinoma: in vivo and in vitro explorations for clinical trial. Int J Gynecol Cancer. 2013;23:1210-1218.

19. White DA, Camus P, Endo M, et al. Noninfectious pneumonitis after everolimus therapy for advanced renal cell carcinoma. Am J Respir Crit Care Med. 2010;182:396-403.

20. National Cancer Institute (2009). Common Terminology Criteria for Adverse Events v4.0. NCI, NIH, DHHS. Available from: https://wiki. nci.nih.gov/display/VKC/Common+Terminology+Criteria+for+Adverse+Events+FAQ\#CommonTerminologyCriteriaforAdverseEventsFA Q-WhatistheofficialreferencecitationforCTCAEv40. Accessed May 29, 2009. 
OncoTargets and Therapy

\section{Publish your work in this journal}

OncoTargets and Therapy is an international, peer-reviewed, open access journal focusing on the pathological basis of all cancers, potential targets for therapy and treatment protocols employed to improve the management of cancer patients. The journal also focuses on the impact of management programs and new therapeutic agents and protocols on

patient perspectives such as quality of life, adherence and satisfaction. The manuscript management system is completely online and includes a very quick and fair peer-review system, which is all easy to use. Visit http://www.dovepress.com/testimonials.php to read real quotes from published authors.

Submit your manuscript here: http://www.dovepress.com/oncotargets-and-therapy-journal 